\section{Reliable systematic review of low- carbohydrate diets shows similar weight-loss effects compared with balanced diets and no cardiovascular risk benefits: Response to methodo- logical criticisms}

To the Editor: Harcombe and Noakes ${ }^{[1]}$ have raised some concerns about our systematic review ${ }^{[2]}$ with questions about the protocol, data extraction and statistical analyses, and the findings.

We started our review by writing a protocol, and defining the question, eligibility criteria and subgroups. ${ }^{[2]}$ This is standard evidence synthesis practice to avoid post-hoc alterations that can create bias. As there are numerous definitions of low-carbohydrate diets, we defined our dietary eligibility criteria by drawing on online advocacy information, ${ }^{[3-6]}$ our structured summary of 50 existing systematic reviews, ${ }^{[2]}$ and national macronutrient recommendations (Australia, USA, Nordic countries, Europe). ${ }^{[7-11]}$ We stated clearly in the paper that we did not intend to investigate macronutrient quality, such as dietary saturated fat content. The subgroups intended were predefined, as protocols should differentiate qualitatively different interventions to help data interpretation and to explore heterogeneity in meta-analysis. ${ }^{[12]}$ Harcombe and Noakes ${ }^{[1]}$ note that one included trial was a duplicate of another trial already included. As the publications did not reference each other, we have subsequently notified the journal editors of this duplication, and have also carried out a sensitivity analysis excluding the duplicate. This made no material difference to the effect estimate.

Harcombe and Noakes ${ }^{[1]}$ criticise various specifics in our data extraction and their comments suggest they did not refer to our protocol, and show lack of understanding of current methods in evidence synthesis. We used data from intention-to-treat analyses (and only if not reported, we used data from per-protocol analyses), and did not report values the wrong way around. Data in the De Luis trials ${ }^{[13,14]}$ can be meta-analysed - there is no problem with combining change and end values in a meta-analysis of randomised controlled trials. ${ }^{[12]}$ Harcombe and Noakes ${ }^{\text {[1] }}$ use of standardised mean difference (SMD) is inappropriate as all trials reported weight in the same unit (kilograms). SMDs are functionally unclear and the meaning of differences and measures of variance have limited interpretability. ${ }^{[15]}$ Harcombe and Noakes ${ }^{[1]}$ use the SMD and various post-hoc adjustments to produce a metaanalysis, at an unspecified time point, on one outcome. They then use statistical significance to claim that low-carbohydrate diets produce greater weight loss. Concluding material benefit based on statistical tests of significance with clinically unimportant effects, rather than on the size of the effect, is a common mistake. Our results show that the estimated average weight loss after 3 6 months in overweight and obese non-diabetics in 13 individual trials ranged from a loss of $2.65-10.20 \mathrm{~kg}$ in people randomised to low-carbohydrate diets, and ranged from a loss of $2.65-9.40 \mathrm{~kg}$ with isoenergetic balanced diets. After 3 - 6 months, the average difference in weight loss between the dietary groups was $780 \mathrm{~g}$ (adjusted from $740 \mathrm{~g}$ in our PLoS One ${ }^{[2]}$ article following exclusion of the duplicate publication), a clinically unimportant difference, ${ }^{[16]}$ as was the average difference of $480 \mathrm{~g}$ after 1 - 2 years. Harcombe and Noakes ${ }^{[1]}$ do not provide clear methods, rationale and time points for their partial re-analysis.

We welcome scrutiny and comments. Having considered these carefully, we stand by our analysis and results. We also report that in overweight and obese adults randomised to low-carbohydrate diets or iso-energetic balanced diets, there is probably little or no clinically important difference in average changes in cardiovascular risk factors for up to 2 years. ${ }^{[2]}$

Declarations. Our review ${ }^{[2]}$ was funded by the South African Medical Research Council, and the Effective Health Care Research Consortium (funded by UKaid from the UK Government Department for International Development). Authors (CEN, AS, TY, PG, JV) of this letter work for a charity committed to using scientifically defensible methods to prepare and update systematic reviews of the effects of health interventions. None of the authors are financially conflicted.

\section{E Naude, A Schoonees}

Centre for Evidence-based Health Care, Department of Global Health, Faculty of Medicine and Health Sciences, Stellenbosch University, Cape Town, South Africa cenaude@sun.ac.za

\section{Senekal}

Division of Human Nutrition, Department of Human Biology, Faculty of Health Sciences, University of Cape Town, South Africa

\section{P Garner}

Centre for Evidence Synthesis, Department of Clinical Sciences, Liverpool School of Tropical Medicine, Liverpool, UK

\section{T Young, J Volmink}

Centre for Evidence-based Health Care, Department of Global Health, Faculty of Medicine and Health Sciences, Stellenbosch University, Cape Town, South Africa; Cochrane South Africa, South African Medical Research Council, Cape Town, South Africa

1. Harcombe Z Noakes TD. The universities of Stellenbosch/Cape Town low-carbohydrate diet review: Mistake or mischief? S Afr Med J 2016;106(12):1179-1182. https://doi.org/10.7196/samj.2016.v106. Mistake or

2. Naude CE, Schoonees A, Young T, Senekal M, Garner P, Volmink J. Low carbohydrate versus Naude $\mathrm{CE}$, Schoonees A, Young T, Senekal M, Garner P, Volmink J. Low carbohydrate versus
isoenergetic balanced diets for reducing weight and cardiovascular risk: A systematic review and metaanalysis. PLoS One 2014;9(7):e100652. https://doi.org/10.1371/journal.pone.0100652

3. Atkins Nutritionals. New Atkins. Atkins Nutritionals, 2011. http://sa.atkins.com/ (accessed 10 Augus 2012)

4. WebMD. The Atkins Diet. WebMD, 2012. http://www.webmd.com/diet/atkins-diet-what-it-is (accessed 10 August 2012)

Zone Labs. Dr Sears Zone Diet. Zone Labs Inc., 2012. http://www.zonediet.com/ (accessed 10 August 2012).

Barnett J. The Zone Diet Explained. Crossfit Impulse, 2009. http://crossfitimpulse.com/the-zone-dietexplained-edited (accessed 10 August 2012).

7. Institute of Medicine Food and Nutrition Board. Dietary Reference Intakes for Energy, Carbohydrate, Fiber, Fat, Fatty Acids, Cholesterol, Protein, and Amino Acids (Macronutrients). Washington, DC: National Academies Press, 2005.

8. Australian National Health and Medical Research Council and the New Zealand Ministry of Health. Nutrient Reference Values for Australia and New Zealand: Including Recommended Dietary Intakes. Nutrient Reference Values for Australia and New Zealand: Including Recommended Dietary Intakes.
Canberra: Australian National Health and Medical Research Council and the New Zealand Ministry of Health, 2006.

of Health, 2006 .
9. Becker W, Lyhne N, Pedersen AN, et al. Nordic Nutrition Recommendations 2004 integrating nutrition and physical activity. Scand J Nutr 2004;48(4):178-187. https://dx.do org/ $10.1080 \% 2 \mathrm{~F} 1102680410003794$

0. Nordic Council of Ministers. Nordic Nutrition Recommendations 2004. Copenhagen: Nordic Council of Ministers, 2004.

1. EFSA Panel on Dietetic Products Nutrition and Allergies (NDA). Dietary Reference Values. Parma: European Food Safety Authority (EFSA), 2010.

2. Higgins D, Green S, eds. Cochrane Handbook for Systematic Reviews of Interventions Version 5.1.1 (updated March 2011). London: John Wiley \& Sons Ltd, 2011.

13. De Luis DA, Aller R, Izaola O, et al. Evaluation of weight loss and adipocytokines levels after two hypocaloric diets with different macronutrient distribution in obese subjects with $r s 9939609$ gene hypocaloric diets with different macronutrient distribution in obese subjects with $r s 9939$
variant. Diabetes Metab Res Rev 2012;28(8):663-668. https://dx.doi.org/10.1002/dmrr.2323

14. De Luis DA, Sagrado MG, Conde R, Aller R, Izaola $O$. The effects of two different hypocaloric diets on glucagon-like peptide 1 in obese adults, relation with insulin response after weight loss. J Diabetes
on Complications 2009;23(4):239-243. https://dx.doi.org/10.1016/j.jdiacomp.2007.12.006

15. Egger M, Smith GD, Altman D. Systematic Reviews in Health Care: Meta-Analysis in Context. 2008. http://olabout.wiley.com/WileyCDA/Section/id-397493.html (accessed 20 January 2017).

16. Jensen MD, Ryan DH, Apovian CM, et al. $2013 \mathrm{AHA} / \mathrm{ACC} / \mathrm{TOS}$ guideline for the management of overweight and obesity in adults: A report of the American College of Cardiology/American Heart Association Task Force on practice guidelines and the obesity society. Circulation 2014;129(25 Suppl 2):S102-S138. https://dx.doi.org/10.1161/01.cir.0000437739.71477.e

S Afr Med J 2017;107(3):170. DOI:10.7196/SAMJ.2017.v107i3.12382 